\title{
Implementation of Activity-Based Costing Method in Calculating Production Cost of Coffee Powder Manufacturing Company In Aceh
}

\author{
Fazli Syam BZ $^{1}$, Iskandarsyah ${ }^{2}$, Dinaroe $^{3}$, Muhammad Muharrozaq ${ }^{4}$ \\ ${ }^{1,3,4}$ Accounting Major, Faculty of Economic and Business, Syiah Kuala University \\ ${ }^{2}$ Management Major, Faculty of Economic and Business, Syiah Kuala University
}

\{fazlisyambz@feb.unsyiah.ac.id ${ }^{1}$ \}

\begin{abstract}
The purpose of this research is to study how the calculation and comparing the application of the traditional system method with the Activity Based Costing system method in the coffee manufacturing industry in Aceh. The result of the calculation can be concluded that the comparison cost between the calculation of the cost of goods produced by the traditional system and the calculation of the price of staple goods production has difference price amount in PT. Indo Cufco about Rp.603/kg (undercost), CV. Kupi Lampineung Rp.4.004/kg (undercost), UD. Kupi Teungku Aceh Rp.60,42/kg (overcost), UD. Degood Gayo Coffee Rp.3.042,6/kg (overcost), and UD. Raya Coffee Arabica Rp.9/kg (undercost).
\end{abstract}

Keywords: Cost of Goods Manufactured, Traditional Method, Activity Based Costing

\section{Introduction}

The industry is one of the drivers of the world economic turnaround. The industry is required to maintain the life-sustainability of its business in order to operate in accordance with the objectives set by the company and always generate profits for its owners. The development of the powder processing industry in Aceh province increasingly rapidly. The production of coffee commodities in Aceh Province is huge, with a total area 121,226 $\mathrm{Ha}$, and producing about 46,828 tons per year. The processing of coffee powder using modern technology becomes one of the efficiency indicators and can improve the quality of the production process. Technological developments also have a complex impact on the industry. With the utilization of these technologies resulted in increased operating costs generated by the company that will impact on the high cost of production.

Calculation of manufacturing cost of the product is all costs used to process raw materials to be finished goods in a certain period of time either fixed or variable costs. The inaccuracy in the calculation of cost of goods manufactured has an adverse effect on the company, because the cost of production serves as the basis for setting the selling price and profit, as a tool to measure the efficiency of the production process implementation as well as the basis for decision making for the management of the company. Therefore, there is a new method in the calculation of the cost of production known as activity-based costing (ABC).

Purpose of the research is to find out the calculation and comparison of cost of production in the coffee processing industry in Aceh Province using traditional system and activity-based costing.

\section{Literature Review}

\subsection{Management Accounting and Cost Accounting}

The definition of management accounting by Horngren, Datar, \& Rajan [1] is the process of identification, measurement and accumulation, analysis and preparation, interpretation, and communication of information that helps each executive to meet organizational goals. Management accounting also includes preparing financial statements for non-management groups such as shareholders, creditors, regulatory agencies. financial and nonfinancial means used by management to make decisions.

While, Horngren, Datar, \& Foster [2] states "Cost Accounting provides the information needed for management accounting and financial accounting. Cost accounting measures and reports any financial and nonfinancial information related to the cost or utilization of resources within an organization". While the definition of costs according to Bustami \& Nurlela [3] cost is the sacrifice of economic resources as measured in units of 
money that have occurred or are likely to occur to achieve certain goals. In addition, Mardiasmo [4] defined manufacturing cost as "the manufactured cost of a product or service is an accumulation of the costs imposed on products or services produced by the company".

\subsection{Traditional System Method vs Activity-Based Costing}

Hansen \& Mowen [5] state that the traditional system is a cost accounting system which assumes that all are classified as fixed or variable related to changes in units or volumes of manufactured products. While activitybased costing according to Raiborn \& Kinney [6] is a cost accounting system that focuses on organizational activities and collection of costs based on the underlying nature of the underlying level of some predetermined overhead and then calculated using a variety of cost drivers in the activities of an organization.

\subsection{Previous Research}

The results of research by Rahmaji [7] entitled "Implementation of Activity Based Costing System to Determine the Cost of Products of PT. Celebes Mina Pratama". PT. Celebes Mina Pratama is a company that produces 3 kinds of product ikan kayu, hana katsuo and fish meal. The research concludes that with activity based costing system able to give a calculation of the cost of production more accurate. The results showed that activitybased costing system when compared with traditional methods then gives greater results. The difference that occurs due to the overhead cost of each product. The differences of this research is the object of research by Rahmaji on fish processing industry, while in research conducted by researchers in the coffee powder processing industry. The similarity of research conducted by Rahmaji with this research is on the subject of research. The company under study still uses Traditional System in calculating the cost of production so it is necessary to do research by using activity based costing system to evaluate the accuracy of calculation done at this time.

The result of the research of Suratinoyo [8] entitled "Application of ABC system for determining the cost of goods manufactured in Build Wenang Beverage". PT. Build Wenang Manado is a manufacturing company engaged in the manufacture of soft drinks. The calculation of the cost of production using the ABC method when compared with the method used by the company there is a difference of Rp.416.242.174 where the total cost of production using the $\mathrm{ABC}$ method is Rp.41.667.875.470. When compared with the traditional system of Rp.42.129.053.094. The differences of this research are the object of his research where Suratinoyo in the manufacture of soft drinks, while researchers in the coffee powder processing industry. The equation of research is to have similarity in using the traditional system to charge product cost so that need to be done by using an activity based costing system to calculate the cost of goods production.

The results of Rotikan [9], entitled "Application of activity-based costing method in determining the cost of production at PT. Tropica Cocoprima ". PT. Tropica Cocoprima is a company engaged in the production of coconut flour. The research yields the conclusion that calculation of the cost of production by ABC method shows undercost condition for ordinary coconut flour product and overcost condition for fine coconut flour.

The differences of this research are that in research undertaken by Rotikan choose coconut flour processing company, where researchers using coffee powder processing company as the subject of his research. The similarity of this research is to have similarities in using Activity-based costing to perform calculations and evaluate the accuracy of the calculations performed today.

\section{Research Methods}

\subsection{Subject and Object of Research}

The research subjects in this research are five coffee processing industry units in Aceh Province (PT Indo Cufco, CV Kupi Lampineung, UD Coffee Tgk Aceh, UD Degood Gayo Coffee, UD Raya Coffee Arabica). The object of research in this research is the data related to the determination of the cost of production. The data consists of: (1) Qualitative data, is data in the form of letters, pictures, diagrams and so forth (not numbers) that describe something or words. In this case, the required data is data about the history of PT. Indo Cufco, CV. Kupi Lampineung, UD. Coffee Tgk. Aceh, UD. Degood Gayo Coffee, UD. Raya Coffee Arabica and the development of these companies, company location, organizational structure, marketing area, production system, etc. (2) Quantitative data, is data in the form of numbers or data that can be calculated by unit count. These data are all 
data related to the production of PT. Indo Cufco, CV. Kupi Lampineung, UD. Coffee Tgk. Aceh, UD. Degood Gayo Coffee, UD. Raya Coffee Arabica in 2016.

\subsection{Operational Definition of Research Variable}

Operational Definition of Research Variable of this research is: (1) Cost of production is all costs used to process raw materials to be finished goods within a certain period of time. (2) The traditional system is a system of determining the cost of production that uses the basis of charging costs in accordance with changes in unit or volume of products produced. (3) Activity-based costing is a cost calculation that emphasizes activities that use more cost driver to measure the resources used by the product more accurately and relevant.

\subsection{Research Design, Data Collection Method and Data Analysis Method}

This research is descriptive research. Descriptive research aims to describe the facts that currently apply. Descriptive research is categorized in the study of the object of past and present variables and describes the variables being studied [10].

Data collection conducted in this research is field study. Field study is a data collection steps taken author directly from PT. Indo Cufco, CV. Kupi Lampineung, UD. Coffee Tgk. Aceh, UD. Degood Gayo Coffee, UD. Raya Coffee Arabica which became the object of this research by:

1. Interviews with the company manager, this interview is a data collection technique that is done with parts that concerned and directly involved with the discussed and associated with the data required.

2. Direct observation of the work system, especially related to the activity-based costing process.

3. Observing procedures or policies implemented by the company.

Data analysis techniques used to calculate the cost of production with Activity-based costing are as follows:

1. Calculating the cost of production with the traditional system.

2. Calculating the cost of production using Activity-based costing, with the following ways:

a. Identifying activity to each level activity (Unit, Batch, Product, and Facility).

b. Determining the homogenous cost pool.

c. Determining the pool rate per units of each cost pool.

d. Allocating pool rate based on cost driver has consumed by each level activity.

e. Arranged the cost of the product by activity-based costing method.

3. Compare the calculation of the cost of production based on traditional system and activity-based costing then calculate the difference.

4. Analyzing a more precise system in determining the cost of production in PT. Indo Cufco, CV. Kupi Lampineung, UD. Coffee Tgk. Aceh, UD. Degood Gayo Coffee, UD. Raya Coffee Arabika.

\section{Result and Discussion}

ABC implementation study on 5 coffee powder processing companies in Aceh Province with different types of products, and different production capacity, in order to obtain various results from each type of product. At PT. Indo Cufco there are 4 types of products (arabica coffee specialty, longberry / peaberry, Luwak, and wine), CV. Kupi Lampineung Utama there are 3 types of products (robusta coffee, arabica coffee, and robusta jagung), UD. Kupi Teungku Aceh has 2 types of products (super robusta coffee and standard robusta coffee), UD. Degood Gayo Coffee has 5 types of products (arabica specialty coffee, long berry, peaberry, Luwak, and wine), and UD. Raya Coffee Arabica produces 2 types of products (specialty arabica and roasted bean coffee). There are several stages in the processing of coffee beans. The first stage, freshly harvested coffee beans were washed and stripped of the outer shell using a depulper machine, clean coffee beans were dried until the humidity was reduced by $30 \%$, after the dry coffee beans were stripped from the coffee beans using a huller machine, the next stage was dried again to get the coffee beans with $12 \%$ moisture content, the last stage is sorting the beans by type and quality.

The dried green beans are then roasted using a roasting machine until they reach the desired level of maturity; there are two commonly used roasting tools, automatic machines, using heat power from gas fuel, and manuals are still done traditionally using fire heat from firewood. After the roasting process is complete then the coffee 
beans are smoothed using a grinding machine to obtain coffee powder and then packed to maintain the taste quality of the coffee into several packing sizes. Powdered coffee ready for market.

The process of processing the coffee beans is not difficult, but requires a lot of manpower if still done manually, the company conducted research has combined with the use of production machinery to support production efficiency and suppress the use of labor, so the five companies have met the criteria for the calculation of price principal production using activity-based costing system.

The calculation of the cost of goods manufactured using activity-based costing system specifies the calculation of overhead costs into 4 activity levels and charges the cost according to the capacity of each product produced. Activity-based costing not only to charge the cost of accurate but also as a tool of cost control, from every activity we do will find out how much the cost incurred. The traditional method of calculation cannot be performed cost control because all costs are charged with the production unit.

Companies that do research still use the calculation of the traditional model, because the application is general and the calculation is simple. But responses from company owners assessed the activity-based costing system is helpful to companies in controlling costs because the activity-based costing system details the calculation of factory overhead cost according to the capacity of each product. So inflate the cost can be easily traced and taken control steps.

\subsection{Specific Data}

The production data of PT. Indo Cufco, CV. Kupi Lampineung, UD. Coffee Tgk. Aceh, UD. Degood Gayo Coffee, and UD. Raya Coffee Arabica in 2016 is presented in Table $1-5$ as follows:

Table 1. Production Data of PT Indo Cufco in the Year 2016

Product Type $\quad$ Production Unit (Kg) $\quad$ Cost Of Good Sold (Rp) $\quad$ Labour Cost (Rp)

\begin{tabular}{lrrr}
\hline Specialty & 5.000 & 1.000 .000 .000 & 85.200 .000 \\
\hline Longberry/ Peaberry & 2.500 & 620.500 .000 & 42.600 .000 \\
\hline Wine & 100 & 40.000 .000 & 2.840 .000 \\
\hline Luwak & 700 & 245.000 .000 & 11.360 .000 \\
\hline Total & 8.300 & 1.910 .000 .000 & 142.000 .000 \\
& Source: PT Indo Cufco & &
\end{tabular}

Table 2. Production Data of CV Kupi Lampineung in the Year 2016

\begin{tabular}{cccc} 
Product Type & Production Unit (Kg) & Cost Of Good Sold (Rp) & Labour Cost (Rp) \\
\hline Robusta (Original) & 972 & 29.191 .000 & 3.575 .000 \\
\hline Arabica (Original) & 1.450 & 115.999 .000 & 5.200 .000 \\
\hline $\begin{array}{c}\text { Robusta (Mixed with } \\
\text { Corn) }\end{array}$ & 6.217 & 162.892 .500 & 23.725 .000 \\
\hline Total & $\begin{array}{c}.639 \\
\text { Source: CV Kupi Lampineung }\end{array}$ & 32.500 .000 \\
& \multicolumn{3}{l}{$\begin{array}{c}308.012 .500 \\
\end{array}$}
\end{tabular}

Table 3. Production Data of UD Kopi Teungku Aceh in the Year 2016

Product Type $\quad$ Production Unit (Kg) Cost Of Good Sold (Rp) Labour Cost (Rp)

\begin{tabular}{lcrr}
\hline Super & 1.000 & 45.000 .000 & 6.338 .028 \\
\hline Standard & 70.000 & 210.000 .000 & 443.661 .972 \\
\hline Total & 71.000 & 255.000 .000 & 450.000 .000
\end{tabular}


Table 4. Production Data of UD Degood Gayo in the Year 2016

\section{Product Type $\quad$ Production Unit (Kg) Cost Of Good Sold (Rp) $\quad$ Labour Cost (Rp)}

\begin{tabular}{lcrr}
\hline Specialty & 1.000 & 200.000 .000 & 37.800 .000 \\
\hline Longberry & 500 & 75.000 .000 & 18.900 .000 \\
\hline Peaberry & 300 & 54.000 .000 & 10.800 .000 \\
\hline Wine & 70 & 28.000 .000 & 3.600 .000 \\
\hline Luwak & 500 & 500.000 .000 & 18.900 .000 \\
\hline Total & 2.370 & 875.000 .000 & 90.000 .000
\end{tabular}

Table 5. Production Data of UD Raya Coffee Arabica in the Year 2016

Product Type
Production Unit (Kg)
Cost Of Good Sold (Rp)

\begin{tabular}{lccc}
\hline Roasted Bean & 1.500 & 157.500 .000 & 14.760 .000 \\
\hline Specialty & 2.100 & 241.500 .000 & 21.240 .000 \\
\hline Total & 3.600 & 399.000 .000 & 36.000 .000
\end{tabular}

Source: UD Raya Coffee Arabica

PT. Indo Cufco, CV. Kupi Lampineung, UD. Kopi Tgk. Aceh, UD. Degood Gayo Coffee, dan UD. Raya Coffee Arabika has overhead costs of production used in 2016 to produce the above products is presented in table 6 as follows:

Table 6. Overhead of the Year 2016 (in Rp)

\begin{tabular}{lrrrrr}
\multicolumn{1}{c}{ Cost } & PT Indo Cufco & $\begin{array}{c}\text { CV Kupi } \\
\text { Lampineung }\end{array}$ & $\begin{array}{c}\text { UD Kupi } \\
\text { Teungku Aceh }\end{array}$ & $\begin{array}{c}\text { UD Degood } \\
\text { Gayo }\end{array}$ & $\begin{array}{c}\text { UD Raya } \\
\text { Coffee } \\
\text { Arabica }\end{array}$ \\
\hline Electricity & 23.985 .450 & 9.700 .000 & 12.000 .000 & 54.000 .000 & 8.400 .000 \\
\hline Vehicle Fuel & 5.000 .000 & 3.800 .000 & 5.000 .000 & 1.500 .000 & 1.700 .000 \\
\hline Machine Fuel & 1.200 .000 & 20.000 .000 & 325.000 & 500.000 & 504.000 \\
\hline Vehicle Sparepart & 3.000 .000 & 500.000 & 400.000 & 5.000 .000 & 300.000 \\
\hline Vehicle Maintenance & 2.400 .000 & 300.000 & 1.000 .000 & 3.000 .000 & 750.000 \\
\hline Machine Maintenance & 500.000 & 200.000 & 300.000 & 3.000 .000 & 800.000 \\
\hline Pickup Cost & 0 & 700.000 & 0 & 0 & 0 \\
\hline Employee Salary & 0 & 0 & 40.000 .000 & 0 & 0 \\
\hline Factory Equipment & 2.000 .000 & 2.000 .000 & 200.000 & 5.000 .000 & 200.000 \\
\hline Cleaning Service & $1.500,000$ & 0 & 0 & 300.000 & 500.000 \\
\hline Employee Training & 4.500 .000 & 0 & 0 & 2.000 .000 & 0 \\
\hline Fix Assets Maintenance & 2.000 .000 & 200.000 & 2.000 .000 & 5.000 .000 & 0 \\
\hline Labor Assurance & 12.000 .000 & 0 & 0 & 12.000 .000 & 0 \\
\hline Vehicle Depreciation & 30.000 .000 & 5.595 .000 & 5.000 .000 & 6.000 .000 & 3.333 .000 \\
\hline Machine Depreciation & 23.333 .000 & 16.785 .000 & 1.912 .000 & 4.000 .000 & 2.000 .000 \\
\hline Factory Depreciation & 5.428 .000 & 9.000 .000 & 7.500 .000 & 2.000 .000 & 1.666 .000 \\
\hline Packaging & 50.000 .000 & 1.500 .000 & 36.000 .000 & 200.000 .000 & 20.000 .000 \\
\hline Shipping Cost & 80.000 .000 & 0 & 0 & 0 & 0 \\
\hline Marketing & 0 & 0 & 150.000 & 10.000 .000 & 1.000 .000 \\
\hline Total & 246.846 .450 & 70.280 .000 & 111.787 .000 & 313.300 .000 & 41.153 .000 \\
& Source: Secondary Data Processed & & & \\
& & & & & 0
\end{tabular}

In addition to the above data, other data used to support the implementation of Activity-Based Costing System, among others:

1. Total electricity consumption

2. Number of hours of the inspection

3. Area of the area used 
The quantity of the data can be presented in table 7 to table 11 as follows:

Table 7. Cost Driver of PT Indo Cufco

\begin{tabular}{|c|c|c|c|c|c|}
\hline \multirow{2}{*}{ Cost Driver } & \multicolumn{4}{|c|}{ Product Variants } & \multirow{2}{*}{ Total } \\
\hline & Specialty & Longberry/ Peaberry & Wine & Luwak & \\
\hline Total unit production $(\mathrm{kg})$ & 5.000 & 2.500 & 100 & 700 & 8.300 \\
\hline Total KWH & 9.810 & 4.905 & 327 & 1.308 & 16.350 \\
\hline Total production hours & 1.725 & 863 & 58 & 230 & 2.876 \\
\hline Area width $\left(\mathrm{m}^{2}\right)$ & 1.080 & $\begin{array}{c}540 \\
\text { Source: PT Indo Cu }\end{array}$ & 36 & 144 & 1.800 \\
\hline
\end{tabular}

Table 8. Cost Driver of CV Kupi Lampineung

\begin{tabular}{|c|c|c|c|c|}
\hline \multirow[b]{2}{*}{ Cost Driver } & \multicolumn{3}{|c|}{ Product Variants } & \multirow[b]{2}{*}{ Total } \\
\hline & Robusta (Original) & Arabica (Original) & $\begin{array}{l}\text { Robusta (Mixed with } \\
\text { Corn) }\end{array}$ & \\
\hline Total unit production $(\mathrm{kg})$ & 972 & 1.450 & 6.217 & 8.639 \\
\hline Total KWH & 735,7 & $1.070,3$ & 4.882 & 6.689 \\
\hline Total production hours & 592 & 861 & 3.927 & 5.380 \\
\hline Area width $\left(\mathrm{m}^{2}\right)$ & 165 & 240 & 1.095 & 1.500 \\
\hline
\end{tabular}

Table 9. Cost Driver of UD Kopi Teungku Aceh

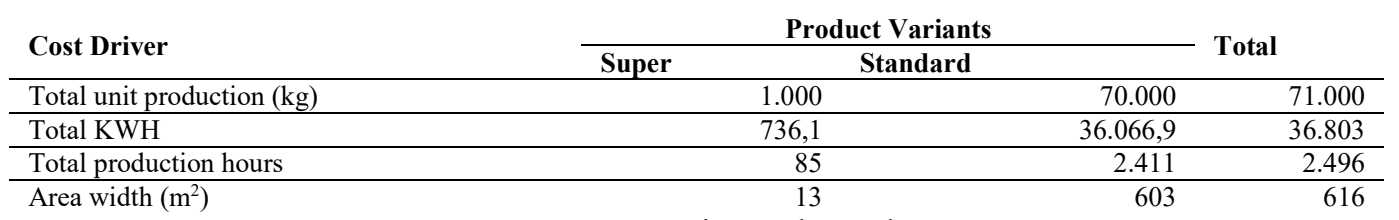

Source: UD Kopi Teungku Aceh

Table 10. Cost Driver of UD. Degood Gayo Coffee

\begin{tabular}{|c|c|c|c|c|c|c|}
\hline \multirow{2}{*}{ Cost Driver } & \multicolumn{5}{|c|}{ Product Variants } & \multirow{2}{*}{ Total } \\
\hline & Specialty & Longberry & Peaberry & Wine & Luwak & \\
\hline Total unit production $(\mathrm{kg})$ & 1.000 & 500 & 300 & 70 & 500 & 2.370 \\
\hline Total KWH & 3.434 & 1.717 & 2.453 & 327 & 1.717 & 8.178 \\
\hline Total production hours & 819 & 409 & 234 & 78 & 409 & 1.950 \\
\hline Area width $\left(\mathrm{m}^{2}\right)$ & 1.050 & 525 & 300 & 100 & 525 & 2.500 \\
\hline
\end{tabular}

Table 11. Cost Driver of UD Raya Coffee Arabica

\begin{tabular}{lrrr}
\multirow{2}{*}{ Cost Driver } & \multicolumn{2}{c}{ Product Variants } & \multirow{2}{*}{ Total } \\
\cline { 2 - 4 } & Roasted Bean & Specialty & 2.100 \\
\hline Total unit production $(\mathrm{kg})$ & 1.500 & 3.377 & 3.600 \\
\hline Total KWH & 2.347 & 1.275 & 2.160 \\
\hline Total production hours & 885 & 236 & 400
\end{tabular}

\subsection{Discussion}

Source: UD Raya Coffee Arabica

\subsubsection{Calculation cost of the product by traditional methods}

The calculation cost of the product by traditional methods is presented in Table $12-16$ as follows. 
Table 12. Production cost calculation with the traditional method of PT Indo Cufco

Product 1 - Specialty

\begin{tabular}{|c|c|c|c|}
\hline Cost Element & Total Cost (Rp) & Quantity (Kg) & Cost per Unit (Rp) \\
\hline Primary cost & 1.085 .200 .000 & 5.000 & 217.040 \\
\hline Overhead cost $=29.740,5 \times 5.000$ & 145.432 .801 & 5.000 & $29.085,5$ \\
\hline Total & & & 246.127 \\
\hline \multicolumn{4}{|l|}{ Product 2 - Longberry/Peaberry } \\
\hline Cost Element & Total Cost (Rp) & Quantity (Kg) & Cost per Unit (Rp) \\
\hline Primary cost & 663.100 .000 & 2.500 & 265.240 \\
\hline Overhead cost $=29.740,5 \times 2.500$ & 72.716 .250 & 2.500 & $29.085,5$ \\
\hline Total & & & 29.325 \\
\hline \multicolumn{4}{|l|}{ Product 3 - Wine } \\
\hline Cost Element & Total Cost (Rp) & Quantity (Kg) & Cost per Unit (Rp) \\
\hline Primary cost & 42.840 .000 & 100 & 428.400 \\
\hline Overhead cost $=29.740,5 \times 100$ & 2.908 .650 & 100 & $29.085,5$ \\
\hline Total & & & 457.485 \\
\hline \multicolumn{4}{|l|}{ Product 4- Luwak } \\
\hline Cost Element & Total Cost (Rp) & Quantity (Kg) & Cost per Unit (Rp) \\
\hline Primary cost & 256.360 .000 & 700 & $366.228,5$ \\
\hline Overhead cost $=29.740,5 \times 700$ & 20.360 .200 & 700 & $29.085,5$ \\
\hline
\end{tabular}

Source: Secondary Data Processed

Table 13. Production cost calculation with the traditional method of CV Kupi Lampineung

Product 1 - Robusta (original)

\begin{tabular}{|c|c|c|c|}
\hline Cost Element & Total Cost (Rp) & Quantity (Kg) & Cost per Unit (Rp) \\
\hline Primary cost & 32.696 .000 & 972 & $33.637,8$ \\
\hline Overhead cost $=8.135,2 \times 972$ & 7.907 .415 & 972 & $8.135,2$ \\
\hline Total & & & 41.773 \\
\hline \multicolumn{4}{|l|}{ Product 2 - Arabica (original) } \\
\hline Cost Element & Total Cost (Rp) & Quantity (Kg) & Cost per Unit (Rp) \\
\hline Primary cost & 121.199 .000 & 1.450 & $83.585,5$ \\
\hline Overhead cost $=8.135,2 \times 1.450$ & 11.796 .040 & 1.450 & $8.135,2$ \\
\hline Total & & & $91.720,7$ \\
\hline \multicolumn{4}{|c|}{ Product 3 - Robusta (mixed with corn) } \\
\hline Cost Element & Total Cost (Rp) & Quantity (Kg) & Cost per Unit (Rp) \\
\hline Primary cost & 186.617 .000 & 6.217 & $30.017,2$ \\
\hline Overhead cost $=8.135,2 \times 6.217$ & 50.575 .295 & 6.217 & $8.135,2$ \\
\hline Total & & & $38.152,4$ \\
\hline
\end{tabular}

Source: Secondary Data Processed

Table 14. Production cost calculation with the traditional method of UD Kupi Teungku Aceh

Product 1 - Super

\begin{tabular}{|c|c|c|c|}
\hline Cost Element & Total Cost (Rp) & Quantity (Kg) & Cost per Unit (Rp) \\
\hline Primary cost & 51.338 .028 & 1.000 & $51.338,02$ \\
\hline Overhead cost $=4.412,67 \times 1.000$ & 4.412 .676 & 1.000 & $4.412,67$ \\
\hline Total & & & $\mathbf{5 5 . 7 5 0 , 7 0}$ \\
\hline \multicolumn{4}{|l|}{ Product 2 - Standard } \\
\hline Cost Element & Total Cost (Rp) & Quantity (Kg) & Cost per Unit (Rp) \\
\hline Primary cost & 653.661 .972 & 70.000 & $9.338,028$ \\
\hline Overhead cost $=4.412,67 \times 70.000$ & 308.887 .320 & 70.000 & $4.412,67$ \\
\hline Total & & & $13.750,70$ \\
\hline
\end{tabular}


Table 15. Production cost calculation with the traditional method of UD Degood Gayo Coffee

Product 1 - Specialty

\begin{tabular}{|c|c|c|c|}
\hline Cost Element & Total Cost (Rp) & Quantity (Kg) & Cost per Unit (Rp) \\
\hline Primary cost & 237.800 .000 & 1.000 & 237.800 \\
\hline Overhead cost $=47.167,5 \times 1.000$ & 47.167 .510 & 1.000 & $47.167,5$ \\
\hline Total & & & 284.967 \\
\hline \multicolumn{4}{|l|}{ Product 2 - Longberry } \\
\hline Cost Element & Total Cost (Rp) & Quantity (Kg) & Cost per Unit (Rp) \\
\hline Primary cost & 93.900 .000 & 500 & 187.800 \\
\hline Overhead cost $=47.167,5 \times 500$ & 23.583 .750 & 500 & $47.167,5$ \\
\hline Total & & & 234.967 \\
\hline \multicolumn{4}{|l|}{ Product 3 - Pieberry } \\
\hline Cost Element & Total Cost (Rp) & Quantity (Kg) & Cost per Unit (Rp) \\
\hline Primary cost & 64.800 .000 & 300 & 216.000 \\
\hline Overhead cost $=47.167,5 \times 300$ & 14.150 .250 & 300 & $47.167,5$ \\
\hline Total & & & 263.167 \\
\hline \multicolumn{4}{|l|}{ Product 4- Wine } \\
\hline Cost Element & Total Cost (Rp) & Quantity (Kg) & Cost per Unit (Rp) \\
\hline Primary cost & 31.600 .000 & 70 & $451.428,5$ \\
\hline Overhead cost $=47.167,5 \times 70$ & 3.301 .690 & 70 & $47.167,5$ \\
\hline Total & & & 498.596 \\
\hline \multicolumn{4}{|l|}{ Product 5- Luwak } \\
\hline Cost Element & Total Cost (Rp) & Quantity (Kg) & Cost per Unit (Rp) \\
\hline Primary cost & 518.900 .000 & 500 & $366.228,5$ \\
\hline Overhead cost $=47.167,5 \times 500$ & 23.583 .750 & 500 & $47.167,5$ \\
\hline Total & & & 1.084 .967 \\
\hline
\end{tabular}

Table 16. Production cost calculation with the traditional method of UD Raya Coffee Arabica Product 1 - Roasted Bean

\begin{tabular}{|c|c|c|c|}
\hline Cost Element & Total Cost (Rp) & Quantity (Kg) & Cost per Unit (Rp) \\
\hline Primary cost & 172.260 .000 & 1.500 & 114.840 \\
\hline Overhead cost $11.431 \times 1.500$ & 17.147 .083 & 1.500 & 11.431 \\
\hline Total & & & 126.271 \\
\hline \multicolumn{4}{|l|}{ Product 2 - Specialty } \\
\hline Cost Element & Total Cost (Rp) & Quantity (Kg) & Cost per Unit (Rp) \\
\hline Primary cost & 262.740 .000 & 2.100 & 125.114 \\
\hline Overhead cost $11.431 \times 2.100$ & 24.005 .100 & 2.100 & 11.431 \\
\hline Total & & & 136.545 \\
\hline
\end{tabular}

\subsubsection{Calculation cost of the product by Activity Based Costing}

\subsubsection{The First Stage Procedure}

The first stage determines the cost of production based on the activity-based costing system is to trace the cost of the Source power to the activities that consume it. This stage can be seen in Table 17 as follows:

Table 17. The classification of costs into various activities in the year 2016

\begin{tabular}{clrrrrr}
$\begin{array}{c}\text { Activity } \\
\text { Level }\end{array}$ & \multicolumn{1}{c}{ Cost } & $\begin{array}{c}\text { PT Indo } \\
\text { Cufco }\end{array}$ & $\begin{array}{c}\text { CV Kupi } \\
\text { Lampineung }\end{array}$ & $\begin{array}{c}\text { UD Kupi } \\
\text { Teungku } \\
\text { Aceh }\end{array}$ & $\begin{array}{c}\text { UD Degood } \\
\text { Gayo }\end{array}$ & $\begin{array}{c}\text { UD Raya } \\
\text { Coffee } \\
\text { Arabica }\end{array}$ \\
\hline \multirow{2}{*}{ Unit } & Electricity & 23.985 .450 & 9.700 .000 & 12.000 .000 & 54.000 .000 & 8.400 .000 \\
\cline { 2 - 7 } & Vehicle Fuel & 5.000 .000 & 3.800 .000 & 5.000 .000 & 1.500 .000 & 1.700 .000 \\
\cline { 2 - 7 } & Vehicle Sparepart & 3.000 .000 & 500.000 & 400.000 & 5.000 .000 & 300.000
\end{tabular}




\begin{tabular}{|c|c|c|c|c|c|c|}
\hline & $\begin{array}{l}\text { Vehicle } \\
\text { Maintenance }\end{array}$ & 2.400 .000 & 300.000 & 1.000 .000 & 3.000 .000 & 750.000 \\
\hline & $\begin{array}{l}\text { Vehicle } \\
\text { Depreciation }\end{array}$ & 30.000 .000 & 5.595 .000 & 5.000 .000 & 6.000 .000 & 3.333 .000 \\
\hline \multirow{7}{*}{ Batch } & Machine Fuel & 1.200 .000 & 20.000 .000 & 325.000 & 500.000 & 504.000 \\
\hline & $\begin{array}{l}\text { Machine } \\
\text { Maintenance }\end{array}$ & 500.000 & 200.000 & 300.000 & 3.000 .000 & 800.000 \\
\hline & Pickup Cost & 0 & 700.000 & 0 & 0 & 0 \\
\hline & Employee Salary & 0 & 0 & 40.000 .000 & 0 & 0 \\
\hline & Labor Assurance & 12.000 .000 & 0 & 0 & 12.000 .000 & 0 \\
\hline & Employee Training & 4.500 .000 & 0 & 0 & 2.000 .000 & 0 \\
\hline & $\begin{array}{l}\text { Machine } \\
\text { Depreciation }\end{array}$ & 23.333 .000 & 16.785 .000 & 1.912 .000 & 4.000 .000 & 2.000 .000 \\
\hline \multirow{4}{*}{ Facility } & Factory Equipment & 2.000 .000 & 2.000 .000 & 200.000 & 5.000 .000 & 200.000 \\
\hline & Cleaning Service & $1.500,000$ & 0 & 0 & 300.000 & 500.000 \\
\hline & $\begin{array}{l}\text { Fix Assets } \\
\text { Maintenance }\end{array}$ & 2.000 .000 & 200.000 & 2.000 .000 & 5.000 .000 & 0 \\
\hline & $\begin{array}{l}\text { Factory } \\
\text { Depreciation }\end{array}$ & 5.428 .000 & 9.000 .000 & 7.500 .000 & 2.000 .000 & 1.666 .000 \\
\hline \multirow{4}{*}{ Product } & Packaging & 50.000 .000 & 1.500 .000 & 36.000 .000 & 200.000 .000 & 20.000 .000 \\
\hline & Shipping Cost & 80.000 .000 & 0 & 0 & 0 & 0 \\
\hline & Marketing & 0 & 0 & 150.000 & 10.000 .000 & 1.000 .000 \\
\hline & Total & 246.846 .450 & 70.280 .000 & 111.787 .000 & 313.300 .000 & 41.153 .000 \\
\hline
\end{tabular}

Source: Secondary Data Processed

After determining the homo- geneous cost pool, then determine the rate per unit cost driver. Pool rate at PT. Indo Cufco, CV. Kupi Lampineung, UD. Degood Gayo Coffee, UD. Coffee Tgk Aceh, UD. Raya Coffee Arabica in 2016 can be seen in Table 18 to table 22 as follows:

Table 18. Pool rate activity of PT Indo Cufco

\begin{tabular}{|c|c|c|}
\hline Cost pool unit level & Overhead & Amount (Rp) \\
\hline Cost pool 1 & Electricity cost & 23.985 .450 \\
\hline Total cost & & 23.985 .450 \\
\hline Total KWH (KWH) & & 16.350 \\
\hline Pool rate 1 & & $1.467,14$ \\
\hline Cost pool unit level & Overhead & Amount (Rp) \\
\hline \multirow{4}{*}{ Cost pool 2} & Vehicle fuel cost & 5.000 .000 \\
\hline & Vehicle spare parts cost & 3.000 .000 \\
\hline & Vehicle maintenance cost & 2.400 .000 \\
\hline & Vehicle depreciation & 30.000 .000 \\
\hline Total cost & & 40.400 .000 \\
\hline Total production unit (Unit) & & 8.300 \\
\hline Pool rate 2 & & $4.867,5$ \\
\hline Cost pool batch level & Overhead & Amount (Rp) \\
\hline \multirow{3}{*}{ Cost pool 3} & Machine fuel cost & 1.200 .000 \\
\hline & Machine maintenance cost & 500.000 \\
\hline & Machine depreciation & 23.333 .000 \\
\hline Total cost & & 25.033 .000 \\
\hline Operate hours (hour) & & 2.876 \\
\hline Pool rate 3 & & 8.704 \\
\hline Cost pool batch level & Overhead & Amount (Rp) \\
\hline \multirow{2}{*}{ Cost pool 4} & Employee training cost & $4.500,000$ \\
\hline & Labor assurance cost & 12.000 .000 \\
\hline Total cost & & 16.500 .000 \\
\hline Operate hours (hour) & & 2.375 \\
\hline Pool rate 4 & & 6.947 \\
\hline Cost pool product level & Overhead & Amount (Rp) \\
\hline \multirow{2}{*}{ Cost pool 5} & Packaging cost & 50.000 .000 \\
\hline & Distribution cost & 80.000 .000 \\
\hline
\end{tabular}


Total cost

130.000 .000

Product unit (unit)

8.300

Pool rate 5

15.663

\begin{tabular}{llr}
\hline \multirow{2}{*}{ Cost pool facility level } & \multicolumn{1}{c}{ Overhead } & Amount (Rp) \\
\hline & & 2.000 .000 \\
\cline { 2 - 3 } Cost pool 6 & Factory equipment cost & 1.500 .000 \\
\cline { 2 - 3 } & Sanitation cost & 2.000 .000 \\
\cline { 2 - 3 } & Fix asset maintenance & 5.428 .000 \\
\cline { 2 - 3 } & Building depreciation & 10.928 .000 \\
Total cost & & 1.800 \\
Area width $\left(\mathrm{m}^{2}\right)$ & & 6.071
\end{tabular}

Source: Secondary Data Processed

Table 19. Pool rate activity of CV Kupi Lampineung

\begin{tabular}{|c|c|c|}
\hline Cost pool unit level & Overhead & Amount (Rp) \\
\hline Cost pool 1 & Electricity cost & 9.700 .000 \\
\hline Total cost & & 9.700 .000 \\
\hline Total KWH (KWH) & & 6.689 \\
\hline Pool rate 1 & & $1.450,14$ \\
\hline Cost pool unit level & Overhead & Amount (Rp) \\
\hline \multirow{4}{*}{ Cost pool 2} & Vehicle fuel cost & 3.800 .000 \\
\hline & Vehicle spare parts cost & 500.000 \\
\hline & Vehicle maintenance cost & 300.000 \\
\hline & Vehicle depreciation & 5.595 .000 \\
\hline Total cost & & 10.195 .000 \\
\hline Total production unit (unit) & & 8.639 \\
\hline Pool rate 2 & & $1.180,11$ \\
\hline Cost pool batch level & Overhead & Amount (Rp) \\
\hline \multirow{3}{*}{ Cost pool 3} & Machine fuel cost & 20.000 .000 \\
\hline & Machine maintenance cost & 200.000 \\
\hline & Machine depreciation & 16.785 .000 \\
\hline Total cost & & 36.985 .000 \\
\hline Operate hours (hour) & & 5.380 \\
\hline Pool rate 3 & & $6.874,53$ \\
\hline Cost pool product level & Overhead & Amount (Rp) \\
\hline Cost pool 4 & Packaging cost & 1.500 .000 \\
\hline Total cost & & 1.500 .000 \\
\hline Product unit (unit) & & 8.639 \\
\hline Pool rate 4 & & 173,63 \\
\hline Cost pool facility level & Overhead & Amount (Rp) \\
\hline \multirow{3}{*}{ Cost pool 5} & Factory equipment cost & 2.000 .000 \\
\hline & Fix asset maintenance & 200.000 \\
\hline & Building depreciation & 9.000 .000 \\
\hline Total cost & & 11.200 .000 \\
\hline Area width $\left(\mathrm{m}^{2}\right)$ & & 1.500 \\
\hline Pool rate 5 & & $7.466,6$ \\
\hline
\end{tabular}

Source: Secondary Data Processed

Table 20. Pool rate activity of UD Kupi Teungku Aceh

Cost pool unit level

\begin{tabular}{llr} 
Cost pool unit level & Overhead & Amount (Rp) \\
\hline Cost pool 1 & Electricity cost & 54.000 .000 \\
\hline Total cost & & 54.000 .000 \\
Total KWH $(\mathrm{KWH})$ & & 36.803 \\
Pool rate 1 & \multicolumn{1}{c}{ Overhead } & $1.467,27$ \\
\hline Cost pool unit level & \multicolumn{1}{c}{. } & Amount (Rp) \\
\hline \multirow{4}{*}{ Cost pool 2 } & \multicolumn{1}{c}{ Vehicle fuel cost } & 5.500 .000 \\
\cline { 2 - 3 } & Vehicle spare parts cost & 3.000 .000 \\
\cline { 2 - 3 } & Vehicle maintenance cost & 6.000 .000 \\
\cline { 2 - 3 } & Vehicle depreciation & 15.500 .000 \\
\hline Total cost & & 71.000 \\
Total production unit (unit) & & 218,30 \\
Pool rate 2 & &
\end{tabular}


Cost pool batch level

Amount (Rp)

\begin{tabular}{|c|c|c|}
\hline \multirow{3}{*}{ Cost pool 3} & Machine fuel cost & 500.000 \\
\hline & Machine maintenance cost & 3.000 .000 \\
\hline & Machine depreciation & 4.000 .000 \\
\hline Total cost & & 7.500 .000 \\
\hline Operate hours (hour) & & 17.752 \\
\hline Pool rate 3 & & 422,48 \\
\hline Cost pool batch level & Overhead & Amount (Rp) \\
\hline \multirow{2}{*}{ Cost pool 4} & Employee training cost & $2.000,000$ \\
\hline & Labor assurance cost & 12.000 .000 \\
\hline Total cost & & 14.000 .000 \\
\hline Operate hours (hour) & & 38.040 \\
\hline Pool rate 4 & & 368,03 \\
\hline Cost pool product level & Overhead & Amount (Rp) \\
\hline \multirow{2}{*}{ Cost pool 5} & Packaging cost & 200.000 .000 \\
\hline & Distribution cost & 10.000 .000 \\
\hline Total cost & & 210.000 .000 \\
\hline Product unit (unit) & & 71.000 \\
\hline Pool rate 5 & & $2.957,74$ \\
\hline Cost pool facility level & Overhead & Amount (Rp) \\
\hline \multirow{4}{*}{ Cost pool 6} & Factory equipment cost & 5.000 .000 \\
\hline & Sanitation cost & 300.000 \\
\hline & Fix asset maintenance & 5.000 .000 \\
\hline & Building depreciation & 2.000 .000 \\
\hline Total cost & & 12.300 .000 \\
\hline Area width $\left(\mathrm{m}^{2}\right)$ & & 2.106 \\
\hline Pool rate 6 & & $5.840,45$ \\
\hline
\end{tabular}

Source: Secondary Data Processed

Table 21. Pool rate activity of UD Degood Gayo

\begin{tabular}{|c|c|c|}
\hline Cost pool unit level & Overhead & Amount (Rp) \\
\hline Cost pool 1 & Electricity cost & 12.000 .000 \\
\hline Total cost & & 12.000 .000 \\
\hline Total KWH (KWH) & & 8.178 \\
\hline Pool rate 1 & & $1.467,14$ \\
\hline Cost pool unit level & Overhead & Amount (Rp) \\
\hline \multirow{4}{*}{ Cost pool 2} & Vehicle fuel cost & 5.000 .000 \\
\hline & Vehicle spare parts cost & 400.000 \\
\hline & Vehicle maintenance cost & 1.000 .000 \\
\hline & Vehicle depreciation & 5.000 .000 \\
\hline Total cost & & 11.400 .000 \\
\hline Total production unit (unit) & & 2.370 \\
\hline Pool rate 2 & & 4.810 \\
\hline Cost pool batch level & Overhead & Amount (Rp) \\
\hline \multirow{4}{*}{ Cost pool 3} & Machine fuel cost & 325.000 \\
\hline & Machine maintenance cost & 300.000 \\
\hline & Machine depreciation & 1.912 .000 \\
\hline & Employee Salary & 40.000 .000 \\
\hline Total cost & & 42.537 .000 \\
\hline Operate hours (hour) & & 1.950 \\
\hline Pool rate 3 & & 21.814 \\
\hline Cost pool product level & Overhead & Amount (Rp) \\
\hline \multirow{2}{*}{ Cost pool 4} & Packaging cost & 36.000 .000 \\
\hline & Marketing cost & 150.000 \\
\hline Total cost & & 36.150 .000 \\
\hline Product unit (unit) & & 2.370 \\
\hline Pool rate 4 & & 15.253 \\
\hline
\end{tabular}




\begin{tabular}{llr} 
& Factory equipment cost & 200.000 \\
\cline { 2 - 3 } Cost pool 5 & Fix asset maintenance & 2.000 .000 \\
\cline { 2 - 3 } & Building depreciation & 7.500 .000 \\
\hline Total cost & & 9.700 .000 \\
Area width $\left(\mathrm{m}^{2}\right)$ & & 2.500 \\
Pool rate 5 & & 3.880
\end{tabular}

Table 22. Pool rate activity of UD Raya Coffee Arabica

\begin{tabular}{|c|c|c|}
\hline Cost pool unit level & Overhead & Amount (Rp) \\
\hline Cost pool 1 & Electricity cost & 8.400 .000 \\
\hline Total cost & & 8.400 .000 \\
\hline Total KWH & & 5.725 \\
\hline Pool rate 1 & & $1.467,14$ \\
\hline Cost pool unit level & Overhead & Amount (Rp) \\
\hline \multirow{4}{*}{ Cost pool 2} & Vehicle fuel cost & 1.700 .000 \\
\hline & Vehicle spare parts cost & 300.000 \\
\hline & Vehicle maintenance cost & 750.000 \\
\hline & Vehicle depreciation & 3.333 .000 \\
\hline Total cost & & 6.083 .000 \\
\hline Total production unit & & 3.600 \\
\hline Pool rate 2 & & 1.689 \\
\hline Cost pool batch level & Overhead & Amount (Rp) \\
\hline \multirow{3}{*}{ Cost pool 3} & Machine fuel cost & 504.000 \\
\hline & Machine maintenance cost & 800.000 \\
\hline & Machine depreciation & 2.000 .000 \\
\hline Total cost & & 3.304 .000 \\
\hline Operate hours & & 2.160 \\
\hline Pool rate 3 & & 1.530 \\
\hline Cost pool product level & Overhead & Amount (Rp) \\
\hline \multirow{2}{*}{ Cost pool 4} & Packaging cost & 20.000 .000 \\
\hline & Distribution cost & 1.000 .000 \\
\hline Total cost & & 21.000 .000 \\
\hline Product unit & & 3.600 \\
\hline Pool rate 4 & & 5.834 \\
\hline Cost pool facility level & Overhead & Amount (Rp) \\
\hline \multirow{3}{*}{ Cost pool 5} & Factory equipment cost & 200.000 \\
\hline & Fix asset maintenance & 500.000 \\
\hline & Building depreciation & 1.666 .000 \\
\hline Total cost & & 2.366 .000 \\
\hline Area width & & 400 \\
\hline Pool rate 5 & & 5.915 \\
\hline
\end{tabular}

\subsubsection{The Secondary Stage Procedure}

The cost of product calculation with activity-based costing system at PT. Indo Cufco, CV. Kupi Lampineung, UD. Degood Gayo Coffee, UD. Coffee Tgk Aceh, UD. Raya Coffee Arabica is presented in Table 23 - 27 as follows:

Table 23. Allocation Overhead by ABC Method in PT Indo Cufco

\begin{tabular}{|c|c|c|c|c|c|c|}
\hline $\begin{array}{c}\text { Activity } \\
\text { Level }\end{array}$ & Cost Driver & Allocation & Specialty & $\begin{array}{c}\text { Longberry/ } \\
\text { Peaberry }\end{array}$ & Wine & Luwak \\
\hline \multirow{8}{*}{ Unit } & \multirow{4}{*}{ KWH } & $1.467 \times 9.810$ & 14.391 .270 & & & \\
\hline & & $1.467 \times 4.905$ & & 7.195 .635 & & \\
\hline & & $1.467 \times 327$ & & & 479.709 & \\
\hline & & $1.467 \times 1.308$ & & & & 1.918 .836 \\
\hline & \multirow{4}{*}{ Total unit } & $4.867,5 \times 5.000$ & 24.337 .500 & & & \\
\hline & & $4.867,5 \times 2.500$ & & 12.167 .500 & & \\
\hline & & $4.867,5 \times 100$ & & & 486.700 & \\
\hline & & $4.867,5 \times 700$ & & & & 3.406 .900 \\
\hline
\end{tabular}




\begin{tabular}{|c|c|c|c|c|c|c|}
\hline \multirow{8}{*}{ Batch } & \multirow{4}{*}{ Operate hours } & $8.704 \times 300$ & 2.611 .200 & & & \\
\hline & & $8.704 \times 150,3$ & & \multicolumn{3}{|l|}{1.308 .211} \\
\hline & & $8.704 \times 10$ & & & 87.040 & \\
\hline & & $8.704 \times 40$ & & & & 348.160 \\
\hline & \multirow{4}{*}{ Operate hours } & $6.947 \times 1.425$ & 9.899 .475 & & & \\
\hline & & $6.947 \times 712,5$ & & 4.949 .737 & & \\
\hline & & $6.947 \times 47,5$ & & & 329.982 & \\
\hline & & $6.947 \times 190$ & & & & 1.319 .930 \\
\hline \multirow{4}{*}{ Product } & \multirow{4}{*}{ Total unit } & $15.663 \times 5.000$ & 78.135 .000 & & & \\
\hline & & $15.663 \times 2.500$ & & 39.157 .500 & & \\
\hline & & $15.663 \times 100$ & & & 1.556 .300 & \\
\hline & & $15.663 \times 700$ & & & & 10.964 .100 \\
\hline \multirow{4}{*}{ Facility } & \multirow{4}{*}{ Area width } & $6.071 \times 1.080$ & 6.556 .680 & & & \\
\hline & & $6.071 \times 540$ & & 3.278 .340 & & \\
\hline & & $6.071 \times 36$ & & & 218.556 & \\
\hline & & $6.071 \times 144$ & & & & 874.224 \\
\hline & Total Ov & & 136.111.125 & 68.056 .923 & 3.158.287 & 18.832 .150 \\
\hline
\end{tabular}

Source: Secondary Data Processed

Table 24. Allocation Overhead by ABC Method in CV Kupi Lampineung

\begin{tabular}{|c|c|c|c|c|c|}
\hline $\begin{array}{c}\text { Activity } \\
\text { Level }\end{array}$ & Cost Driver & Allocation & $\begin{array}{c}\text { Robusta } \\
\text { (original) }\end{array}$ & $\begin{array}{c}\text { Arabica } \\
\text { (original) }\end{array}$ & $\begin{array}{l}\text { Robusta (mixed } \\
\text { with corn) }\end{array}$ \\
\hline \multirow{6}{*}{ Unit } & \multirow{3}{*}{ KWH } & $1.450,14 \times 735,7$ & 1.066 .868 & & \\
\hline & & $1.450,14 \times 1.070,3$ & & $1.552 .084,84$ & \\
\hline & & $1.450,14 \times 4.882$ & & & $7.079 .583,48$ \\
\hline & \multirow{3}{*}{ Total unit } & $1.180,11 \times 972$ & 1.147 .067 & & \\
\hline & & $1.180,11 \times 1.450$ & & $1.711 .159,5$ & \\
\hline & & $1.180,11 \times 6.217$ & & & $7.336 .743,87$ \\
\hline \multirow{3}{*}{ Batch } & \multirow{3}{*}{ Operate hours } & $1.883 \times 207,1$ & $389.969,3$ & & \\
\hline & & $1.883 \times 301,3$ & & 567.348 & \\
\hline & & $1.883 \times 1.374,6$ & & & 2.588 .372 \\
\hline \multirow{3}{*}{ Product } & \multirow{3}{*}{ Total unit } & $173,63 \times 972$ & $168.739,2$ & & \\
\hline & & $173,63 \times 1.450$ & & 251.720 & \\
\hline & & $173,63 \times 6.217$ & & & $1.079 .271,2$ \\
\hline \multirow{3}{*}{ Facility } & \multirow{3}{*}{ Area width } & $7.466,6 \times 165$ & 1.231 .989 & & \\
\hline & & $7.466,6 \times 240$ & & 1.7791 .984 & \\
\hline & & $7.466,6 \times 1.095$ & & & 8.175 .927 \\
\hline & Total Ov & & $4.004 .632,5$ & $5.874 .296,34$ & $26.259 .879,6$ \\
\hline
\end{tabular}

Table 25. Allocation Overhead by ABC Method in UD Kupi Teungku Aceh

\begin{tabular}{|c|c|c|c|c|}
\hline $\begin{array}{c}\text { Activity } \\
\text { Level }\end{array}$ & Cost Driver & Allocation & Super & Standard \\
\hline \multirow{4}{*}{ Unit } & \multirow{2}{*}{ KWH } & $1.407,27 \times 518,35$ & $760.559,40$ & \\
\hline & & $1.407,27 \times 38.284,64$ & & $53.239 .363,7$ \\
\hline & \multirow{2}{*}{ Total unit } & $218,30 \times 1.000$ & 218.300 & \\
\hline & & $218,30 \times 70.000$ & & 15.281 .000 \\
\hline \multirow{4}{*}{ Batch } & \multirow{2}{*}{ Operate hours } & $422,48 \times 250$ & $226.449,28$ & \\
\hline & & $422,48 \times 17.502$ & & $7.394 .244,96$ \\
\hline & \multirow{2}{*}{ Operate hours } & $368,03 \times 536$ & $197.264,08$ & \\
\hline & & $368,03 \times 37.504$ & & $13.802 .597,1$ \\
\hline \multirow{2}{*}{ Product } & \multirow{2}{*}{ Total unit } & $2.957,74 \times 1.000$ & 2.957 .740 & \\
\hline & & $2.957,74 \times 70.000$ & & 207.041 .800 \\
\hline Facility & Area width & $5.840,45 \times 29,66$ & $173.225,90$ & \\
\hline
\end{tabular}


Table 26. Allocation Overhead by ABC Method in UD Degood Gayo

\begin{tabular}{|c|c|c|c|c|c|c|c|}
\hline $\begin{array}{l}\text { Activit } \\
\text { y Level }\end{array}$ & Cost Driver & Allocation & Specialty & Longberry & Peaberry & Wine & Luwak \\
\hline \multirow{10}{*}{ Unit } & \multirow{5}{*}{ KWH } & $1.467 \times 3.434$ & 5.037 .678 & & & & \\
\hline & & $1.467 \times 1.717$ & & 2.518 .839 & & & \\
\hline & & $1.467 \times 2.453$ & & & 3.598 .551 & & \\
\hline & & $1.467 \times 327$ & & & & 479.709 & \\
\hline & & $1.467 \times 1.717$ & & & & & 2.518 .839 \\
\hline & \multirow{5}{*}{ Total unit } & $4.810 \times 1.000$ & 4.810 .000 & & & & \\
\hline & & $4.810 \times 500$ & & 2.405 .000 & & & \\
\hline & & $4.810 \times 300$ & & & 1.443 .000 & & \\
\hline & & $4.810 \times 70$ & & & & 336.700 & \\
\hline & & $4.810 \times 500$ & & & & & 2.405 .000 \\
\hline \multirow{5}{*}{ Batch } & \multirow{5}{*}{ Operate hours } & $21.814 \times 819$ & 17.865 .666 & & & & \\
\hline & & $21.814 \times 409$ & & 8.934 .196 & & & \\
\hline & & $21.814 \times 234$ & & & 5.104 .476 & & \\
\hline & & $21.814 \times 78$ & & & & 1.701 .492 & \\
\hline & & $21.814 \times 409$ & & & & & 8.934 .196 \\
\hline \multirow{5}{*}{ Product } & \multirow{5}{*}{ Total unit } & $15.253 \times 1.000$ & 15.253 .000 & & & & \\
\hline & & $15.253 \times 500$ & & 7.626 .500 & & & \\
\hline & & $15.253 \times 300$ & & & 4.575 .900 & & \\
\hline & & $15.253 \times 70$ & & & & 1.067 .710 & \\
\hline & & $15.253 \times 500$ & & & & & 7.626 .500 \\
\hline \multirow{5}{*}{ Facility } & \multirow{5}{*}{ Area width } & $3.880 \times 1.050$ & 4.074 .000 & & & & \\
\hline & & $3.880 \times 525$ & & 2.037 .000 & & & \\
\hline & & $3.880 \times 300$ & & & 1.164 .000 & & \\
\hline & & $3.880 \times 100$ & & & & 388.000 & \\
\hline & & $3.880 \times 525$ & & & & & 2.037 .000 \\
\hline & Total Overl & & 47.040 .344 & 23.521 .535 & 15.885 .927 & 3.973 .611 & 23.621 .535 \\
\hline
\end{tabular}

Source: Secondary Data Processed

Table 27. Allocation Overhead by ABC Method in UD Raya Coffee Arabica

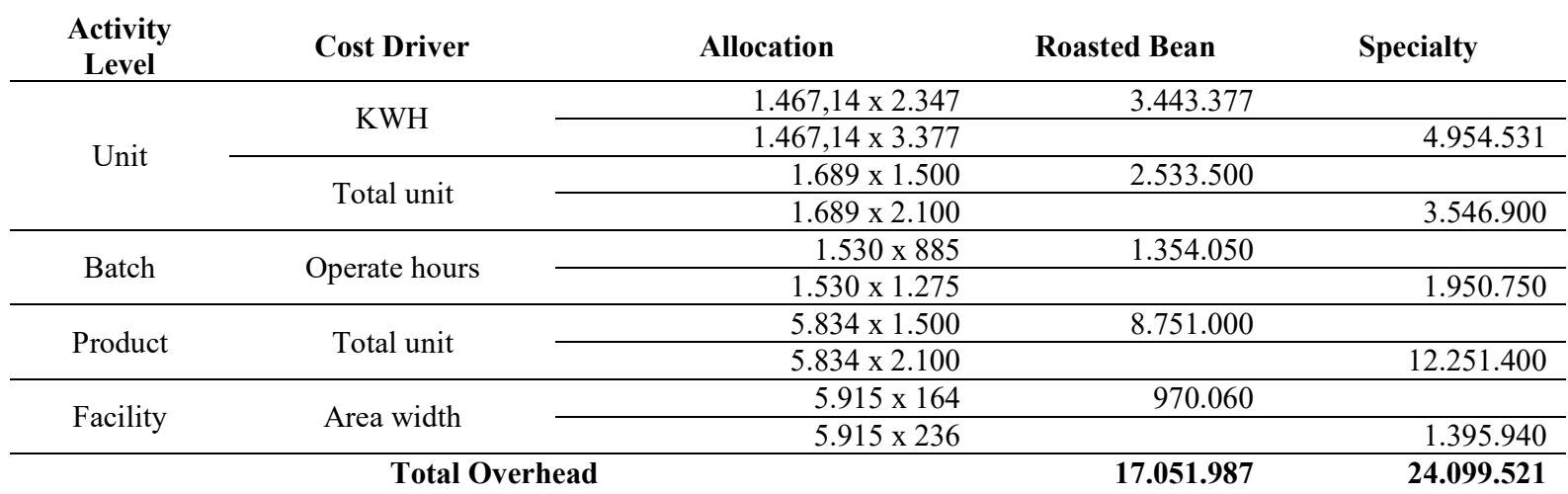

Source: Secondary Data Processed 
The calculation of the cost of production by using the activity-based costing system at PT. Indo Cufco, CV. Kupi Lampineung, UD. Degood Gayo Coffee, UD. Coffee Tgk Aceh, UD. Raya Coffee Arabica in 2016 can be presented in Table 4.28 - 4.32 as follows:

Table 28. Calculation cost of the product by activity-based costing system of PT. Indo Cufco

\begin{tabular}{lrrrr} 
Component & \multicolumn{1}{c}{ Specialty } & Longberry/ Peaberry & \multicolumn{1}{c}{ Wine } & \multicolumn{1}{c}{ Luwak } \\
\hline Raw materials & 1.000 .000 .000 & 620.500 .000 & 40.000 .000 & 245.000 .000 \\
\hline Labor & 85.200 .000 & 42.600 .000 & 2.840 .000 & 11.360 .000 \\
\hline Overhead & 136.111 .125 & 68.056 .923 & 3.158 .287 & 18.832 .150 \\
\hline Cost of product & 1.221 .311 .125 & 731.156 .923 & 45.998 .287 & 100 \\
\hline Unit product $(\mathrm{Kg})$ & 5.000 & 2.500 & $\mathbf{4 5 9 . 9 8 3}$ & 700 \\
\hline Cost per unit & $\mathbf{2 4 4 . 2 6 2}$ & $\mathbf{2 9 2 . 4 6 3}$ & & $\mathbf{3 9 3 . 1 3 1}$ \\
Source: Secondary data processed & & & &
\end{tabular}

Table 29. Calculation cost of the product by activity-based costing system of CV. Kupi Lampineung

\begin{tabular}{lrrr}
\multicolumn{1}{c}{ Component } & Robusta (original) & Arabica (Original) & \multicolumn{1}{c}{ Robusta (mixed with corn) } \\
\hline Raw materials & 29.121 .000 & 115.999 .000 & 162.892 .500 \\
\hline Labor & 3.575 .000 & 5.200 .000 & 23.725 .000 \\
\hline Overhead & 4.004 .632 .5 & $5.874 .296,34$ & $26.259 .879,6$ \\
\hline Cost of product & $36.700 .632,5$ & 127.073 .296 & 212.874 .380 \\
\hline Unit product $(\mathrm{Kg})$ & 972 & 1.450 & 6.217 \\
\hline Cost per unit & $\mathbf{3 7 . 7 5 7 , 8 5}$ & $\mathbf{8 7 . 6 3 6 , 7 5}$ & $\mathbf{3 4 . 2 4 0 , 7}$ \\
Source: Secondary data processed & & &
\end{tabular}

Source: Secondary data processed

Table 30. Calculation cost of the product by activity-based costing system of UD Kupi Teungku Aceh

\begin{tabular}{lrr}
\multicolumn{1}{c}{ Component } & Super & \multicolumn{1}{c}{ Standard } \\
\hline Raw materials & 45.000 .000 & 210.000 .000 \\
\hline Labor & 6.338 .028 & 443.661 .972 \\
\hline Overhead & 4.533 .540 & 308.885 .754 \\
\hline Cost of product & 55.871 .568 & 962.547 .726 \\
\hline Unit product $(\mathrm{Kg})$ & 1.000 & $\mathbf{7 0 . 0 0 0}$ \\
\hline Cost per unit & $\mathbf{5 5 . 8 7 1 , 5 6}$ & $\mathbf{1 3 . 7 5 0 , 6 8}$ \\
Source: Secondary data processed & &
\end{tabular}

Table 31. Calculation cost of the product by activity-based costing system of UD Degood Gayo

\begin{tabular}{lrrrrr} 
Component & \multicolumn{1}{c}{ Specialty } & \multicolumn{1}{c}{ Longberry } & Peaberry & \multicolumn{1}{l}{ Wine } & \multicolumn{1}{c}{ Luwak } \\
\hline Raw materials & 200.000 .000 & 75.000 .000 & 54.000 .000 & 28.000 .000 & 500.000 .000 \\
\hline Labor & 37.800 .000 & 18.900 .000 & 10.800 .000 & 3.600 .000 & 18.900 .000 \\
\hline Overhead & 47.040 .344 & 23.521 .535 & 15.885 .927 & 3.973 .611 & 23.621 .535 \\
\hline Cost of product & 284.840 .344 & 117.421 .535 & 80.685 .927 & 35.573 .611 & 542.521 .535 \\
\hline Unitproduct(Kg) & 1.000 & 500 & 300 & 70 & 500 \\
\hline Cost per unit & $\mathbf{2 8 4 . 8 4 0}$ & $\mathbf{2 3 4 . 8 4 3}$ & $\mathbf{2 6 8 . 9 5 3}$ & $\mathbf{5 0 8 . 1 9 4}$ & $\mathbf{1 . 0 8 5 . 0 4 3}$
\end{tabular}

Source: Secondary data processed

Table 32. Calculation cost of the product by activity-based costing system of UD Raya Coffee Arabica

\begin{tabular}{lrr} 
Component & Roasted Bean & \multicolumn{1}{c}{ Specialty } \\
\hline Raw materials & 157.500 .000 & 241.500 .000 \\
\hline Labor & 14.760 .000 & 21.240 .000 \\
\hline Overhead & 17.051 .987 & 24.099 .521 \\
\hline Cost of product & 189.311 .987 & 286.839 .521 \\
\hline Unit product $(\mathrm{Kg})$ & 1.500 & 2.100 \\
\hline Cost per unit & $\mathbf{1 2 6 . 2 0 8}$ & $\mathbf{1 3 6 . 5 9 0}$ \\
Source: Secondary data processed & &
\end{tabular}




\subsubsection{The Tertiary Stage Procedure}

Comparing traditional systems with activity-based costing system in determining the cost of production. Comparison of cost of production of traditional system with activity-based costing system is presented in Table $33-37$ as follows:

Table 33. Comparison of cost of production by traditional system and activity-based costing system of PT. Indo Cufco

\begin{tabular}{|c|c|c|c|c|}
\hline Method & Specialty (TC/kg) & $\begin{array}{c}\text { Longberry/ } \\
\text { Peaberry (TC/kg) }\end{array}$ & Wine (TC/kg) & Luwak (TC/kg) \\
\hline Traditional & $1.230 .635 .000 /$ & $733.312 .500 /$ & $45.748 .500 /$ & $276.719 .800 /$ \\
\hline System & 246.127 & 293.325 & 457.485 & 395.314 \\
\hline \multirow{2}{*}{ ABC System } & $1.221 .311 .125 /$ & $731.156 .923 /$ & $45.998 .287 /$ & $275.192 .150 /$ \\
\hline & 244.262 & 292.463 & 459.983 & 393.131 \\
\hline \multirow{2}{*}{ Difference } & $9.323 .875 /$ & $2.155 .577 /$ & $-249.787 /$ & $1.527 .650 /$ \\
\hline & 1.865 & 862 & -2.498 & 2.183 \\
\hline
\end{tabular}

Source: Secondary data processed

Table 34. Comparison of cost of production by traditional system and activity based costing system of CV. Kupi Lampineung

\begin{tabular}{lrrr}
\multicolumn{1}{c}{ Method } & $\begin{array}{c}\text { Robusta Original } \\
\text { (TC / kg) }\end{array}$ & $\begin{array}{c}\text { Arabika Original } \\
\text { (TC / kg) }\end{array}$ & $\begin{array}{c}\text { Robusta Mixed with Corn } \\
\text { (TC/ kg) }\end{array}$ \\
\hline Traditional & $40.603 .415 /$ & $132.995 .040 /$ & $237.192 .295 /$ \\
System & 41.773 & $91.720,7$ & $38,152,4$ \\
\hline ABC & $36.700 .632,5 /$ & $127.073 .296 /$ & $212.874 .380 /$ \\
System & $37.757,85$ & $87.636,75$ & $34.240,7$ \\
\hline \multirow{2}{*}{ Difference } & $3.902 .783 /$ & $5.921 .744 /$ & $24.317 .915 /$ \\
& 4.015 & 4.083 & $3.911,5$ \\
\hline Value & & Overcost & Overcost
\end{tabular}

Source: Secondary data processed

Table 35. Comparison of cost of production by traditional system and activity-based costing system of UD Kupi Teungku Aceh

\begin{tabular}{|c|c|c|}
\hline Method & $\begin{array}{c}\text { Super } \\
\text { (TC / kg) }\end{array}$ & $\begin{array}{l}\text { Standard } \\
\text { (TC / kg) }\end{array}$ \\
\hline Traditional & 55.750 .704 & $962.549 .280 /$ \\
\hline System & $55.750,70$ & $13.750,70$ \\
\hline $\mathrm{ABC}$ & $55.871 .568 /$ & $962.547 .725 /$ \\
\hline System & $55.871,56$ & $13.750,68$ \\
\hline Difference & $120.860 /$ & $1.555 /$ \\
\hline Dinerence & 120,86 & 0,02 \\
\hline
\end{tabular}

Source: Secondary data processed

Table 36. Comparison of cost of production by traditional system and activity-based costing system of UD Degood Gayo

\begin{tabular}{|c|c|c|c|c|c|}
\hline Method & Specialty (TC/kg) & $\begin{array}{c}\text { Longberry } \\
\text { (TC/kg) }\end{array}$ & Peaberry (TC/kg) & $\begin{array}{c}\text { Wine } \\
\text { (TC/kg) }\end{array}$ & $\begin{array}{l}\text { Luwak } \\
\text { (TC/kg) }\end{array}$ \\
\hline Traditional & $284.967 .000 /$ & $117.483 .500 /$ & $78.950 .100 /$ & $34.901 .720 /$ & $542.483 .500 /$ \\
\hline System & 284.967 & 234.967 & 263.167 & 498.596 & 1.084 .967 \\
\hline ABC System & $\begin{array}{r}284.840 .000 / \\
284.840\end{array}$ & $\begin{array}{r}117.421 .535 / \\
234.843\end{array}$ & $\begin{array}{r}80.685 .927 / \\
268.953\end{array}$ & $\begin{array}{r}35.573 .611 / \\
508.194\end{array}$ & $\begin{array}{r}542.521 .535 / \\
1.085 .043\end{array}$ \\
\hline
\end{tabular}




\begin{tabular}{lrrrrrr} 
& $126.656 /$ & & $61.965 /$ & $-1.735 .827 /$ & $-671.891 /$ & $-38.053 /$ \\
Difference & 126 & & 123 & $-5.786,09$ & -9.598 & $-76,07$ \\
\hline Value & Overcost & Overcost & & Undercost & Undercost & \multicolumn{3}{c}{ Overcost } & \\
Source: Secondary data processed & & & & & &
\end{tabular}

Table 37. Comparison of cost of production by traditional system and activity based costing system of UD Raya Coffee Arabica

\begin{tabular}{|c|c|c|}
\hline Method & $\begin{array}{c}\text { Super } \\
(\mathrm{TC} / \mathrm{kg})\end{array}$ & $\begin{array}{l}\text { Syandard } \\
\text { (TC / kg) }\end{array}$ \\
\hline Traditional & $189.406 .500 /$ & $286.744 .500 /$ \\
\hline System & 126.271 & 136.545 \\
\hline $\mathrm{ABC}$ & 189.311.987/ & $286.839 .521 /$ \\
\hline System & 126.208 & 136.590 \\
\hline & $94.513 /$ & $-95.021 /$ \\
\hline Difference & 63 & $-45,24$ \\
\hline
\end{tabular}

Source: Secondary data processed

\section{Conclusion, Limitation, And Suggestion}

\subsection{Conclusion}

Based on the results of research and discussion conducted by the author at PT. Indo Cufco, CV. Kupi Lampineung, UD. Degood Gayo Coffee, UD. Coffee Tgk Aceh, UD. Raya Coffee Arabica, it can be concluded as follows:

1. The results of the five study subjects get the result of calculating the cost of production of different activity-based costing, that the company that specifies the complete overhead component of the factory tends to be more efficient when using the cost of production activity-based costing. This is evidenced in the company PT. Indo Cufco and CV. Kupi Lampineung.

2. In a company that incompletely informs the cost component of the factory overhead cost, the cost of production of the traditional method is more efficient.

3. Based on these conclusions, the difference between the cost of production by using traditional system and activity-based costing is caused by the overhead of factory overhead cost for each product. In traditional systems, the cost of each product is only charged to one cost driver only. As a result, there tends to be a distortion in the loading of factory overhead costs. In the activity-based costing method, the factory overhead cost for each product is charged to many cost drivers according to the use of production capacity, so that activity-based costing is able to allocate activity cost to each product type appropriately based on the consumption of each activity.

\subsection{Limitation}

This study has several limitations that can be considered for further research to be refined to obtain better results in the future. Limitations contained in this study include:

1. In this study, the data used is not completely real according to the conditions of consumption Source power companies in production, due to limited access and recording company financial statements have not been maximized.

2. The results of the calculation of the cost of production using this activity-based costing system cannot be generalized to other coffee powder processing industries in Aceh 
Province, because each company has a difference in the component overhead cost of the manufacturer, so not all companies are more efficient using the activity method based costing.

\subsection{Suggestion}

To examine the reference for further research, there are several suggestions that can be put forward, among others:

1. For the PT. Indo Cufco, CV. Kupi Lampineung, UD. Degood Gayo Coffee, UD. Coffee Tgk Aceh, UD. Raya Coffee Arabica

a. Cost of production at PT. Indo Cufco, CV. Kupi Lampineung, UD. Degood Gayo Coffee, UD. Coffee Tgk Aceh, UD. Raya Coffee Arabica with activity-based costing shows results largely higher than the cost of production to the traditional system, but preferably for UD. Degood Gayo Coffee, UD. Coffee Tgk Aceh, UD. Raya Coffee Arabica should provide details of the Overhead Cost of the manufacturer component in accordance with the number of activities according to established standards and reevaluate the system determines the cost of production because it will greatly affect the purchasing power of products on the market.

b. PT. Indo Cufco, CV. Kupi Lampineung, should apply the calculation of the cost of production using activity-based costing method, while the UD. Degood Gayo Coffee, UD. Coffee Tgk Aceh, UD. Raya Coffee Arabica is still efficient if using a traditional system because the calculation of the cost of production is cheaper than the ABC method, so it can compete with the market price. If the company produces an increasingly varied product then the company can adopt an activity-based costing system for more accurate cost loading.

2. For the next researcher

For further research, it is better to use other research objects, apart from manufacturing companies. Researchers may use service companies such as insurance companies, hospitals, hotels or consulting firms to obtain more varied information.

\section{REFERENCES}

[1] C. T. Horngren, S. M. Datar, and M. V. Rajan, Cost accounting: A managerial emphasis, 15th ed. New Jersey: Pearson Education Inc., 2015.

[2] C. T. Horngren, S. M. Datar, and G. M. Foster, Akuntansi biaya: Dengan penekanan manajerial. Dialih bahasakan oleh P. A. Lestari, 12th ed. Jakarta: Erlangga, 2008.

[3] B. Bustami and Nurlela, Akuntansi biaya, 4th ed. Jakarta: Mitra Wacana Media, 2013.

[4] Mardiasmo, Akuntansi biaya: Penentuan harga pokok produksi. Yogyakarta: Andi Offset, 1994.

[5] D. R. Hansen and M. M. Mowen, Managerial accounting: Akuntansi manajerial. Dialih Bahasakan Oleh Deny Arnos Kwary, 8th ed. Jakarta: Salemba Empat, 2009.

[6] C. A. Raiborn and M. R. Kinney, Akuntansi biaya: Dasar dan perkembangan, 7th ed. Jakarta: Salemba Empat, 2011.

[7] D. Rahmaji, "Penerapan activity-based costing system untuk menentukan harga pokok produksi PT. Celebes Mina Pratama," J. EMBA, vol. 1, no. 3, pp. 63-73, 2013.

[8] A. W. Suratinoyo, "Penerapan sistem ABC untuk penentuan harga pokok produksi pada Bangun Wenang Beverage,” J. EMBA, vol. 1, no. 3, pp. 658-668, 2013. 
[9] G. S. Rotikan, "Penerapan metode activity based costing dalam penentuan harga pokok produksi pada PT. Tropica Cocoprima," J. EMBA, vol. 1, no. 3, pp. 1019-1029, 2013.

[10] J. Sarwono, Metode penelitian kuantitatif dan kualitatif. Yogyakarta: Graha Ilmu, 2006. 